Journal for ImmunoTherapy of Cancer

\title{
T-cell lymphoma secondary to checkpoint inhibitor therapy
}

\author{
Kartik Anand (D) , ${ }^{1}$ Joe Ensor, ${ }^{2}$ Sai Ravi Pingali, ${ }^{1}$ Patrick Hwu, ${ }^{3,4}$ Madeleine Duvic, ${ }^{3}$ \\ Stephen Chiang, ${ }^{1}$ Roberto Miranda, ${ }^{3}$ Youli Zu, ${ }^{1}$ Swaminathan Iyer (i) ${ }^{3}$
}

To cite: Anand K, Ensor J, Pingali SR, et al. T-cell lymphoma secondary to checkpoint inhibitor therapy. Journal for ImmunoTherapy of Cancer 2020;8:e000104. doi:10.1136/jitc-2019-000104

- Additional material is published online only. To view please visit the journal online (http://dx.doi.org/10.1136/jitc2019-000104).

Accepted 16 December 2019

Check for updates

(c) Author(s) (or their employer(s)) 2020. Re-use permitted under CC BY-NC. No commercial re-use. See rights and permissions. Published by BMJ.

${ }^{1}$ Houston Methodist Cancer Center, Houston, Texas, USA ${ }^{2}$ Houston Methodist Research Institute, Houston, Texas, USA ${ }^{3}$ University of Texas MD Anderson Cancer Center, Houston, Texas, USA

${ }^{4}$ Division of Cancer Medicine, University of Texas MD Anderson Cancer Center, Houston, TX, USA

Correspondence to Dr Kartik Anand; kartikanand88@gmail.com

Dr Swaminathan lyer; spiyer@mdanderson.org

\begin{abstract}
Background Murine model suggests programmed cell death-1 (PD-1), an immune checkpoint not only plays role in tumor escape but is also a tumor suppressor for T-cells. But until, no reports of secondary T-cell lymphoma postuse of immune checkpoint inhibitors (ICls) has been reported. Herein, we present a hitherto unreported phenomenon of secondary T-cell lymphoma when PD-1 inhibitor was used in a patient diagnosed with a tumor of epithelial origin.
\end{abstract}

Case report A man in mid-70s presented with biopsyproven metastatic tumor of epithelial origin. Patient received carboplatin in combination with paclitaxel for four cycles leading to partial remission. The patient was subsequently switched to pembrolizumab due to persistent disease in the mediastinum. After four cycles of PD-1 inhibitor, patient presented with progression of disease and was diagnosed with biopsy-proven peripheral T-cell lymphoma-not otherwise specified. Based on the reported tumor suppressor function of PD-1 in murine models, we hypothesized that the use of PD-1 inhibitor caused clonal proliferation of abnormal T-cell clone leading to T-cell lymphoma. T-cell receptor (TCR) sequencing was performed by TCR $\beta$ sequencing and T-cell clones from pre-ICI treatment specimen were compared with post-ICI treatment specimens. We show that one of the T-cell clones present in pre-ICI treatment specimen at a low frequency of had massive expansion to become most dominant clone in post-ICl treatment specimens leading to lymphoma. Moreover, targeted exome sequencing revealed a new TET2 mutation in the clone representing the lymphoma. Next, we retrospectively reviewed the Food and Drug Administration (FDA) Adverse Events Reporting System (FAERS), the pharmacovigilance database from 2012 to 2018 to find the reported incidence of this phenomenon and calculated the reporting $O R(R O R)$ for disproportionality analysis for risk of T-cell lymphoma due to checkpoint inhibitors compared with other drugs. In FAERS, the incidence of T-cell lymphoma post-ICls (pembrolizumab, nivolumab and ipilimumab) was found to be $0.02 \%$ with $17 \%$ mortality. The ROR probability of risk of T-cell lymphoma compared with other drugs in pharmacovigilance database was increased at 1.91. Conclusions T-cell lymphoma is a rare sequela of ICls with high mortality. Larger studies with long-term followup of patients receiving ICls is needed.

\section{BACKGROUND}

Immune checkpoint inhibitors (ICIs) targeting programmed cell death-1 (PD-1) or cytotoxic T-lymphocyte-associated protein 4 to overcome tumor immune escape have greatly improved outcomes in many cancer subtypes. ${ }^{12}$ However, ICIs are not without toxicity due to off target effects of immune stimulation known as immune-related adverse events (AEs), ${ }^{3}$ which can be potentially life threatening such as myocarditis. ${ }^{4}$ As ICIs are relatively newer class of antineoplastic drugs full extent of toxicity is not known. Immune checkpoint PD-1 not only plays a role in tumor escape through inactivation of T-cell when bound by its ligand programmed cell death ligand 1/2 (PD-L1/2) on tumor cells through PI3K pathway ${ }^{5}$ but is a tumor suppressor for T-cells itself. Wartewig $e t a t^{6}$ discovered the previously unknown role of PD-1 being tumor suppressor when they investigated in a syngeneic mouse model carrying itk-syk oncogenic driver mutation that treatment with PD-1 inhibitor caused massive proliferation of abnormal T-cells carrying oncogenic driver mutation leading to T-cell lymphogenesis. Until now no case of secondary T-cell lymphoma post-treatment with PD-1 inhibitor has been described. Herein, we present a case of secondary T-cell lymphoma in a patient treated with PD-1 inhibitor for tumor of epithelial origin. We also retrospectively queried FDA Adverse Events Reporting System (FAERS) from 2012 to 2018 to find out incidence of this $\mathrm{AE}$ and performed disproportionality signal analysis using reporting OR (ROR).

\section{CASE PRESENTATION}

A man in mid-70s with history of stage T1 transitional cell carcinoma of bladder 14 years prior (treated at that time with intravesical BCG vaccine and intravesical instillation of doxorubicin for vaccine failure) presented to the hospital with shortness of breath. Complete blood count (CBC) and complete metabolic panel were within normal limits. Workup revealed left-sided pleural effusion (figure 1A). Incisional biopsy of left pleura was performed which on immunohistochemistry (IHC) was positive for cytokeratin (CK) and negative 


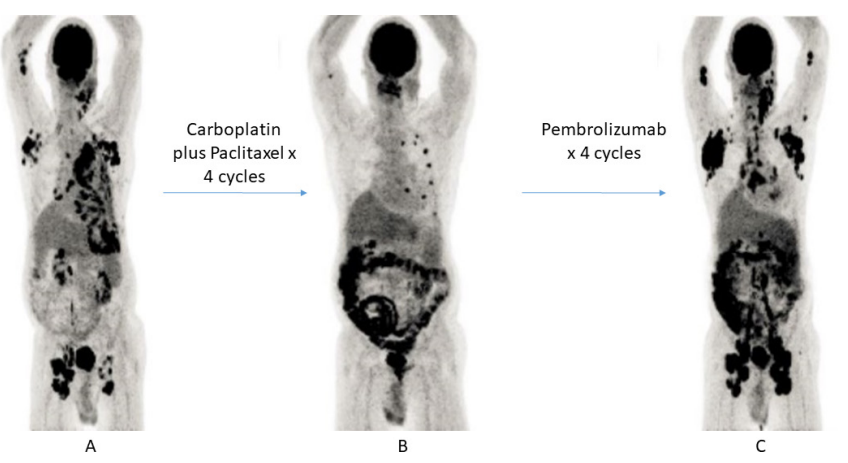

Figure 1 Serial PET scans. (A) Initial PET scan showing increased 18 fludeoxyglucose uptake. (B) PET scan post four cycles of carboplatin plus paclitaxel showing partial remission. (C) PET scan post-PD-1 inhibitor therapy. PD-1, programmed cell death-1.

for CD45 and anaplastic lymphoma kinase (ALK) consistent with undifferentiated carcinoma of epithelial origin (see online supplementary figure 1). PD-L1 staining on the tumor was $5 \%$. Targeted next-generation sequencing (NGS) was performed, which showed low tumor mutation burden of five mutations/megabase with mutation involving TP53 being most predominant; variant allele frequency $(\mathrm{VAF})=31 \%$ (see online supplementary table 1 for all mutations detected). Patient received chemotherapy with standard dose carboplatin in combination with paclitaxel every 3 weeks. After four cycles, the patient had partial response to therapy (figure 1B) and treatment was switched to pembrolizumab (a PD-1 inhibitor) $200 \mathrm{mg}$ intravenously every 3 weeks due to persistent disease in the mediastinum. After four cycles of pembrolizumab, the patient had fever, weight loss along with left cervical, bilateral axillary and inguinal lymphadenopathy (figure 1C). CBC revealed marked lymphocytosis with eosinophilia. Biopsy of left cervical lymph node which on IHC was positive for CD3, CD5, CD30, CD45 and negative for CK, CD20, ALK was consistent with peripheral T-cell lymphoma; not otherwise specified (PTCL;NOS) (online supplementary figure 2). Bone marrow staging biopsy showed the same findings as lymph node confirming Stage IV disease. Targeted exome NGS showed TET2 mutation; VAF $=26 \%$ (c.5127T>A or p.Cys1709Ter, a nonsense mutation in exon 11 of TET2). Unfortunately, the patient developed cerebral venous thrombosis, which led family to decline any further therapy for lymphoma and decided to pursue hospice and patient died shortly thereafter. Based on the reported tumor suppressor function of PD-1 in murine models, we hypothesized that anti PD-1 therapy caused monoclonal expansion of TET2 mutated T-cell clone causing T-cell lymphoma. To prove our hypothesis after obtaining informed consent from the patient family, we performed T-cell receptor (TCR) sequencing (TCR $\beta$ sequencing by Immunoseq assay) and compared pre-ICI to post-ICI biopsy sample. TCR sequencing revealed clonal expansion of T-cell clone (CASTADGSSNTGELFF) from $0.008 \%$ in lung (pre-ICI sample) to $11 \%$ in bone marrow and $41 \%$ in the lymph node (post-ICI samples) which is suspected to

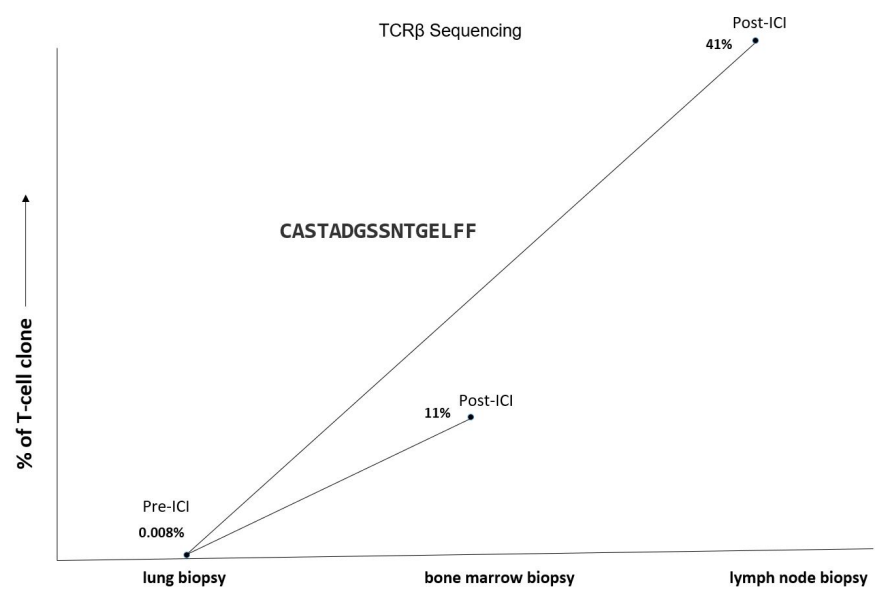

Figure 2 TCR $\beta$ sequencing. T-cell clone by TCR $\beta$ sequencing before $\mathrm{PD}-1$ inhibitor therapy in lung was $0.008 \%$, which expanded to $11 \%$ in bone marrow and $41 \%$ in lymph node (post-treatment samples). Amino acid sequence of T-cell clone was CASTADGSSNTGELFF. ICI, immune checkpoint inhibitor; PD-1, programmed cell death-1; TCR, T-cell receptor.

be the TET2 mutated clone, hence supporting our hypothesis (figure 2) (online supplementary figure 3). Next to find out incidence of this $\mathrm{AE}$, we retrospectively queried FAERS for AEs secondary to ICIs namely 'nivolumab', 'ipilimumab' and 'pembrolizumab' from 1 January 2012 to 31 December 2018. FAERS quarterly files were downloaded and all T-cell-related neoplasm reactions due to ICIs were searched, six were found to be present namely 'T-cell lymphoma', 'mycosis fungoides', 'adult T-cell lymphoma/ leukemia', 'T-cell type acute leukemia', 'anaplastic largecell lymphoma' and 'PTCL unspecified'. To compare risk of T-cell-related neoplasm in ICI treated cases versus cases treated by all other drugs in the database, a disproportionality signal analysis was conducted using ROR. ROR is a measure of the magnitude of association between an exposure to a pharmaceutical and odds of a specific outcome occurring. ${ }^{7}$ It is considered significant when lower limit of $95 \% \mathrm{CI}$ is $>1$. We found 12 cases of T-cell-related neoplasm out of 50445 AEs reported due to ICIs, with incidence of $0.02 \%$ (table 1 ). Seventy-five per cent were men, median age of the whole cohort was 65 years and $50 \%$ were reported in 2018. Half of the cases were secondary to nivolumab, followed by pembrolizumab, nivolumab +ipilimumaband ipilimumab involved in 3, 2 and 1, respectively. Most common indication for ICI use was lung cancer, followed by malignant melanoma, Hodgkin disease, oesophageal carcinoma and renal cancer. Median time to AE was 10.5 months with $17 \%(2 / 12)$ cases with outcome of death. ROR for ICIs causing T-cell lymphoma was elevated at 1.91 (95\% CI 1.08 to 3.37 ) (online supplementary table 2 ). The analysis of FAERS has limitations, this study is retrospective, actual incidence of T-cell lymphoma post-ICI use cannot be determined because it is possible not all the events are reported within FAERS and time of event for all cases was not available. It is critical that any hypotheses generated by 
Table 1 Review of Food and Drug Administration adverse events reporting (pharmacovigilance database)

Total adverse events (nivolumab, 50445 pembrolizumab, ipilimumab)

Total T-cell related neoplasm AE 12

Sex

$\begin{array}{ll}\text { Male } & 8 \\ \text { Female } & 4\end{array}$

Median age, years; no of patients for whom $n=9$ data was available

$\begin{array}{ll}\text { Range (minimum/maximum) } & 65 \text { (51-87) } \\ \text { CPI used } & \\ \text { Nivolumab } & 6 \\ \text { Pembrolizumab } & 3 \\ \text { Nivolumab+ipilimumab } & 2 \\ \quad \text { Ipilimumab } & 1 \\ \text { Indication for CPI use } & \\ \quad \text { Lung cancer } & 5 \\ \quad \text { Malignant melanoma } & 3 \\ \quad \text { Hodgkin disease } & 2 \\ \quad \text { Esophageal carcinoma } & 1 \\ \text { Renal cancer } & 1 \\ \text { Time to AE, months; no of patients for } & \mathrm{n}=7\end{array}$

whom data were available

Range (minimum/maximum) 10.5 (1.33-121.5)

Outcome

$\begin{array}{ll}\text { Death } & 2 \\ \text { Life threatening } & 2 \\ \text { Other outcome } & 8\end{array}$

Year event reported

\begin{tabular}{ll}
2012 & 1 \\
2015 & 1 \\
2016 & 1 \\
2017 & 3 \\
2018 & 6 \\
\hline
\end{tabular}

Country where event occurred

\begin{tabular}{ll} 
USA & 5 \\
Japan & 3 \\
\hline Hong Kong & 1 \\
Spain & 1 \\
Brazil & 1 \\
Canada & 1 \\
\hline
\end{tabular}

$\mathrm{AE}$, adverse event; CPI, checkpoint inhibitor.

using pharmacovigilance databases are validated through prospective studies.

\section{DISCUSSION}

This study reports, to our knowledge, the first report of secondary malignancy with use of ICIs. We report a case of PTCL, NOS in a patient where PD-1 inhibitor was used for undifferentiated carcinoma of epithelial origin. We also review FAERS database to show increased reports of incidence of T-cell-related neoplasm with ICIs, particularly PD-1 inhibitors. TCR signaling on T-cells expressing PD-1 can be inactivated when engaged by its ligand PDL-1/2 on tumor cells through PI3K pathway. ${ }^{5}$ Addition of ICI normally overcomes this PD-1-PD-L1mediated T-cell anergy and promotes T-cell proliferation with enhanced antitumor T-cell responses. Wartewig $e t a t^{6}$ discovered a previously unknown role of PD-1 of being a tumor suppressor. They used a syngeneic mouse model of T-cell lymphoma carrying itk-syk oncogenic driver mutation. In presence of PD-1 expansion of T-cells expressing itk-syk was diminished, while in absence of PD-1 the mice (with biallelic PD-1 deletion) had to be euthanized rapidly due to unregulated expansion and accumulation of T-cells expressing itk-syk. Next they treated syngeneic mice with itk-syk oncogenic driver mutation with PD-1 inhibitor and found massive lymphoproliferation and hyperprogression of lymphoma as in itk-syk carrying mice with PD-1 deletion. Rapid progression of T-cell lymphoma has also been described when nivolumab (a PD-1 inhibitor) was used for adult T-cell lymphoma. ${ }^{8}$ Another clinical trial evaluating pembrolizumab for relapsed/ refractory T-cell lymphoma was stopped prematurely due to futility; at initial restaging scan more than $50 \%$ had disease progression. ${ }^{9}$ T-cell lymphoma as a complication of ICI treatment for other malignancies has not been described. Our patient developed TET2-mutated T-cell lymphoma when pembrolizumab was used to treat the epithelial tumor, closely resembling the process in the itk-syk model. We demonstrate this by finding of massive clonal expansion using the Immunoseq TCR sequencing that tracks the T-cell clone from the lung biopsy specimen at very low frequency to the dominant one in the bone marrow and lymph node. Furthermore, targeted exome NGS revealed a TET2 mutation in exon $11^{10}$ and this was not detected previously on targeted exome NGS in the lung specimen. Mutations in TET2 gene have been reported in higher frequency in T-cell lymphomas, ${ }^{11-13}$ for example, more than one-third of PTCL-NOS cases harbor TET2 mutations. ${ }^{13}$ Studies have shown genomic disruption of TET2 alone is not enough for overt oncogenesis. ${ }^{14}$ Disruption of TET2 can lead to altered differentiation of T-lymphocyte as demonstrated by Fraietta $e t$ $a l,{ }^{15}$ which reported a patient with B cell chronic lymphocytic leukemia treated with anti CD19 chimeric antigen receptor therapy (CAR-T) wherein the CAR-T lentivirus was randomly integrated into the TET2 gene leading to disruption on one allele and patient already had exon 11 mutation on second TET2 allele. They show that TET2 mutation affecting exon 11 only leads to hypofunctioning of gene. They noted that one CAR +T-cells which had this disruption of TET2 gene on both alleles was able to proliferate massively becoming the dominant T-cell clone in the blood leading to durable remission. TET2 disrupted CAR +T-cells were even detected in peripheral circulation 
4 years later with concern of patient developing T-cell malignancy. In our patient, the use of pembrolizumab lead to 'second hit' with the loss of PD-1 tumor suppressor function and the clonal proliferation of this TET2 mutant, culminating in the transformation to the T-cell lymphoma. The observed clinical phenomenon is supported by our query into FAERS, which demonstrates this likelihood of this complication at $0.02 \%$. The ROR for T-cell lymphoma post-ICI use was increased at 1.91 . T-cell lymphoma post ICI is indeed an emerging but a rare complication with a relatively high mortality.

\section{CONCLUSION}

T-cell lymphoma is a rare complication of ICIs with high mortality. Long-term follow-up of patients receiving ICIs particularly PD-1 inhibitors is needed.

\section{Twitter Swaminathan lyer @DrSwami_lyer}

Acknowledgements Authors acknowledge the family of patient for donating tissue for research. The manuscript was previously presented in part as a poster presentation at ASCO-SITC Immuno Oncology symposium in 2019 and KA was awarded ASCO Cancer Conqueror Merit award. We acknowledge grant support (SIDottie and Jimmy Adair Foundation; YZ (PI) and SI (Co-PI)- 5R01CA224304-02 from National Institutes of Health).

Contributors Contribution conceptualisation: KA. SI: methodology. KA, SI and JE: statistical analysis. JE: radiological review. SC: pathological review. YZ: writing original draft preparation. KA and SI. Writing review and editing: all authors.

Funding This study was funded by Houston Methodist Hospital.

Competing interests None declared.

Patient consent for publication Not required.

Provenance and peer review Not commissioned; externally peer reviewed.

Open access This is an open access article distributed in accordance with the Creative Commons Attribution Non Commercial (CC BY-NC 4.0) license, which permits others to distribute, remix, adapt, build upon this work non-commercially, and license their derivative works on different terms, provided the original work is properly cited, appropriate credit is given, any changes made indicated, and the use is non-commercial. See http://creativecommons.org/licenses/by-nc/4.0/.

\section{ORCID iDs}

Kartik Anand http://orcid.org/0000-0003-4480-1443

Swaminathan lyer http://orcid.org/0000-0002-1646-813X

\section{REFERENCES}

1 Reck M, Rodríguez-Abreu D, Robinson AG, et al. Pembrolizumab versus chemotherapy for PD-L1-positive non-small-cell lung cancer. N Engl J Med 2016;375:1823-33.

2 Coit DG, Thompson JA, Albertini MR, et al. Cutaneous melanoma, version 2.2019, NCCN clinical practice guidelines in oncology. J Natl Compr Canc Netw 2019;17:367-402.

3 Brahmer JR, Lacchetti C, Schneider BJ, et al. Management of immune-related adverse events in patients treated with immune checkpoint inhibitor therapy: American Society of clinical oncology clinical practice guideline. J Clin Oncol 2018;36:1714-68.

4 Johnson DB, Balko JM, Compton ML, et al. Fulminant myocarditis with combination immune checkpoint blockade. N Engl J Med 2016;375:1749-55

5 Sharpe AH, Pauken KE. The diverse functions of the PD1 inhibitory pathway. Nat Rev Immunol 2018;18:153-67.

6 Wartewig T, Kurgyis Z, Keppler S, et al. PD-1 is a haploinsufficient suppressor of T cell lymphomagenesis. Nature 2017;552:121-5.

7 Rothman KJ, Lanes S, Sacks ST. The reporting odds ratio and its advantages over the proportional reporting ratio. Pharmacoepidemiol Drug Saf 2004;13:519-23.

8 Ratner L, Waldmann TA, Janakiram M, et al. Rapid progression of adult T-cell leukemia-lymphoma after PD-1 inhibitor therapy. $N$ Engl J Med 2018;378:1947-8.

9 Barta SK, Zain J, MacFarlane AW, et al. Phase II study of the PD-1 inhibitor pembrolizumab for the treatment of relapsed or refractory mature T-cell lymphoma. Clin Lymphoma Myeloma Leuk 2019;19:356-64.

10 Kopanos C, Tsiolkas V, Kouris A, et al. VarSome: the human genomic variant search engine. Bioinformatics 2019;35:1978-80.

11 Couronné L, Bastard C, Bernard OA. Tet2 and Dnmt3a mutations in human T-cell lymphoma. N Engl J Med 2012;366:95-6.

12 Quivoron C, Couronné L, Della Valle V, et al. Tet2 inactivation results in pleiotropic hematopoietic abnormalities in mouse and is a recurrent event during human lymphomagenesis. Cancer Cell 2011;20:25-38.

13 Lemonnier F, Couronné L, Parrens M, et al. Recurrent TET2 mutations in peripheral T-cell lymphomas correlate with TFH-like features and adverse clinical parameters. Blood 2012;120:1466-9.

14 Zang S, Li J, Yang H, et al. Mutations in 5-methylcytosine oxidase TET2 and RhoA cooperatively disrupt T cell homeostasis. J Clin Invest 2017;127:2998-3012.

15 Fraietta JA, Nobles CL, Sammons MA, et al. Disruption of TET2 promotes the therapeutic efficacy of CD19-targeted T cells. Nature 2018;558:307-12. 